Canad. Math. Bull. Vol. 20 (1), 1977

\title{
NOTE ON BURDE'S RATIONAL BIQUADRATIC RECIPROCITY LAW
}

\author{
KENNETH S. WILLIAMS
}

A short proof is given of a biquadratic reciprocity law proved by Burde in 1969 .

Let $p$ and $q$ be primes $\equiv 1(\bmod 4)$ such that $(p \mid q)=(q \mid p)=1$. Then there are integers $a, b, c, d$ with

$$
\begin{array}{lll}
p=a^{2}+b^{2}, & a \equiv 1(\bmod 2), & b \equiv 0(\bmod 2), \\
q=c^{2}+d^{2}, & c \equiv 1(\bmod 2), & d \equiv 0(\bmod 2) .
\end{array}
$$

Set

$$
(p \mid q)_{4}=\left\{\begin{array}{cc}
+1 & \text { if } p \text { a biquadratic residue }(\bmod q), \\
-1, & \text { otherwise }
\end{array}\right.
$$

Burde [2] proved using the law of biquadratic reciprocity that

$$
(p \mid q)_{4}(q \mid p)_{4}=(-1)^{(q-1) / 4}(a d-b c \mid q) .
$$

Lehmer [4, 5] has given two proofs of (1) using results from cyclotomy. In this note we put together two classical results ((2) and (4) below) to give a short proof of (1).

It is easy to show that $( \pm a d \pm b c \mid q)=(a d-b c \mid q)$ for any choice of signs so that (1) is independent of the particular choices made of $a, b, c, d$. We choose $a, b$ to satisfy $a-b+1 \equiv 0(\bmod 4)$ and set $\pi=a+b i$ so that $\pi \bar{\pi}=p$. For any integer $x \neq 0(\bmod p)$ we define a biquadratic character by

$$
(x \mid \pi)_{4}=i^{e} \quad \text { if } \quad x^{(p-1) / 4} \equiv i^{e}(\bmod \pi), \quad 0 \leq e \leq 3 .
$$

The Gauss sum corresponding to this character is

$$
G=\sum_{x=0}^{p-1}(x \mid \pi)_{4} \exp (2 \pi i x / p) .
$$

It is well-known that (see for example [1])

$$
G^{2}=(-1)^{(p-1) / 4} p^{1 / 2} \pi
$$

Raising $G$ to the $q$ th power we obtain by a familiar argument

$$
G^{q} \equiv(q \mid \pi)_{4}^{-1} G \equiv(q \mid p)_{4} G(\bmod q)
$$

that is

$$
G^{q-1} \equiv(q \mid p)_{4}(\bmod q)
$$


Taking the $(q-1) / 2$ th power of (2) and using (3) we obtain

$$
(q \mid p)_{4} \equiv p^{(q-1) / 4} \pi^{(q-1) / 2}(\bmod q) .
$$

or

$$
(p \mid q)_{4}(q \mid p)_{4} \equiv(a+i b)^{(q-1) / 2}(\bmod q) .
$$

It follows from an old result of Dörrie [3] that

$$
(a+i b)^{(q-1) / 2} \equiv(-1)^{(q-1) / 4}(a d-b c \mid q)(\bmod q)
$$

which completes the proof of (1). For completeness we give a proof of (4). We have

$$
d(a+b i) \equiv a d-b c(\bmod c+d i)
$$

so that

$$
(a+b i)^{(q-1) / 2} \equiv(-1)^{(q-1) / 4}(a d-b c \mid q)(\bmod c+d i)
$$

as it is well known that $(d \mid q) \equiv d^{(q-1) / 2} \equiv(-1)^{(q-1) / 4}(\bmod q)$.

Also

$$
d(a+b i) \equiv a d+b c(\bmod c-d i)
$$

so that

(6) $(a+b i)^{(q-1) / 2} \equiv(-1)^{(q-1) / 4}(a d+b c \mid q)$

$$
\equiv(-1)^{(q-1 / 4}(a d-b c \mid q)(\bmod c-d i) .
$$

(4) now follows from (5) and (6).

\section{REFERENCES}

1. P. Bachmann, Die Lehre von der Kreisteilung, Leipzig (1872), equation (9), p. 169.

2. K. Burde, Ein rationales biquadratisches Reziprozitätsgesetz, Jour. reine angew. Math., 235 (1969), 175-184.

3. H. Dörrie, Das quadratische Reciprocitätsgesetz in quadratischen Zahlkörper mit der Classenzahl 1, Gött. Diss., 1898.

4. E. Lehmer, Criteria for cubic and quartic residuacity, Mathematika 5 (1958), 20-29.

5. E. Lehmer, On the quadratic character of some quadratic surds, Jour. reine angew. Math., 250 (1971), 42-48.

CARLETON UNIVERSITY

OtTawa, Canada 